\title{
Closed Mitral Commissurotomy (CMC) at Bangabandhu Sheikh Mujib Medical University - Outcome Study ZERZINA RAHMAN ${ }^{1}$, MOSTAFANURUZZAMAN ${ }^{1}$, NAZIB AHSAN ${ }^{1}$, AYESHA SULTANA ${ }^{1}$, REZWANUL HOQUE ${ }^{2}$, ASLAM HOSSAIN ${ }^{2}$ \\ ${ }^{1}$ Department of Anesthesia (Cardiac Anesthesia wing), Bangabandhu Sheikh Mujib Medical University, Dhaka, Bangladesh, \\ ${ }^{2}$ Deptartment of Cardiac Surgery, Bangabandhu Sheikh Mujib Medical University, Dhaka, Bangladesh
}

Address for correspondence: Dr. Zerzina Rahman, Associated Professor, Department of Anesthesia (Cardiac Anesthesia wing), Bangabandhu Sheikh Mujib Medical University, Dhaka, Bangladesh. E mail - bannya84@gmail.com

\begin{abstract}
:
Mitral stenosis (MS) occurs nearly exclusively as a consequence of rheumatic fever. The rheumatic process evokes the inflammatory changes in the valve endothelium resulting in narrowing of the orifice. Depending upon the severity of the disease, the treatment modality varies and the choice may be either Percutaneous transluminal mitral commissurotomy (PTMC), Closed mitral commissurotomy (CMC), Open mitral commissurotomy or valvuloplasty (OMC) or straight a way mitral valve replacement (MVR) procedure. In this observational study the results of 25 CMC operations done from February '09 through September '09 is presented. A review of results of CMC operations done abroad is also presented. The outcome of this low cost operation for early cases of mitral stenosis is quite satisfactory.
\end{abstract}

Keyword: Rheumatic valvular disease: Closed mitral commissurotomy: immediate outcome

\section{Introduction:}

Mitral stenosis (MS) occurs nearly exclusively as a consequence of rheumatic fever. Thickening of the valve leaflets with commissural fusion and thickening and shortening of the chordae tendinae gradually reduces the size of mitral valve orifice and the efficiency of the left ventricular filling. ${ }^{1}$ These changes decrease the forward output and increase the left atrial and pulmonary venous pressure, leading to congestive heart failure. The development of atrial fibrillation may further decrease ventricular filling and may precipitate symptoms of CHF. The development of pulmonary hypertension may eventually lead to right sided heart failure and functional tricuspid regurgitation. The pathophysiology of mitral stenosis depend on the grading of the stenosis -mild, moderate, severe. Critical stenosis of the valve may occur even 10 to 20 years after initial infection. Symptoms (Fatigue, dyspnoea on exertion, haemoptysis) may be worsened when increased cardiac output is needed, as with pregnancy, illness, anaemia and exercise. Blood stasis in the left atrium is a risk for thrombus formation and systemic embolization.

Depending upon the severity of the disease, the treatment modality varies and the choice may be either Percutaneous transluminal mitral commissurotomy (PTMC), Closed mitral ommissurotomy (CMC), Open mitral commissurotomy or vlavuloplasty (OMC) or straight way mitral valve replacement (MVR) procedure. In BSMMU cardiac centre, closed mitral commissurotomy is a rather common surgery. Most of the patients with rheumatic valvular disease in this country come from the poorer class of the society, who are very prone to develop stenosis, calcification or pulmonary hypertension. Due to low financial status, they approach late for any surgical or invasive procedure and so the complication rate is very high. CMC is a very old technique, questions arise whether this is an outdated surgery for mitral stenosis or not and some of the centers in USA had to wait 21 years to collect 339 patients with mitral stenosis of which 103 were operated with CMC. ${ }^{2}$ But the affected poor patients can not afford the cost of replacement by a mechanical or bioprosthetic valve and the regular follow up for anticoagulant therapy and CMC remains a life saving procedure for them. By this they could earn ten to fifteen years of relatively symptom free life and bail out time to arrange money for future valve replacement procedure. The risk of this operation in expert hand is minimum and the hospital stay period and working period loss are also low. The outcome of open mitral commissurotomy is better than CMC and it provides excellent early and long term results in a selected group of patients. ${ }^{3}$ But some preferred CMC as a safe alternative to OMC \& PTMC. In post-surgical intensive care unit, the immediate outcome of CMC within 3 days of operation can predict also the late results.

\section{Materials \& Methods:}

This was an observational study done in BSMMU cardiac centre on 25 CMC patients, the surgery being done from 
February 2009 through September 2009. Both male and female patients between 18 to 50 years with moderate to severe mitral stenosis were included in the study. Patients with mitral valvular calcification, left atrial thrombus and severe subvalvular changes and more than grade 2 regurgitation were excluded. Patients with organized thrombus in the left atrial appendage were included for CMC. Informed consent was taken from all the patients. Patients' pre-operative data were collected. The surgery $\&$ anesthesia followed the standard technique. Post operative immediate clinical outcome of the patients and at their 3 to 4 day stay in the ICU were recorded.

\section{Results:}

The age range was 18 to 50 years; mean age in male was $31.25 \pm 3.8$ years and the mean age in female was27.47 \pm 4.1 years. The mean body weight was $40 \mathrm{~kg}$ in male and 43.5 $\mathrm{kg}$ in female. Mean height in male was $155.71 \mathrm{~cm}$ and in female was $152.33 \mathrm{~cm}$. Pre operative variables are described in Table-I.The global ejection fraction was maintained in both male and female patient though the average mitral valve orifice areas which are less than $1 \mathrm{~cm}^{2}$ in both group of patients. Organized thrombus was present in left atrial appendages in 2 female patients, one patient was pregnant, 5 patients were more than 40 years and pulmonary hypertension was present in 14 patients. NYHA grading was done which shows grade 4 in $45 \%$ of male and $50 \%$ of female; grade 3 in $45 \%$ of male and $40 \%$ of female and lastly grade 2 in $10 \%$ of both male and female patients.

In male patients $3.50 \mathrm{~cm}$ dilatation was done whereas in female patients $3.25 \mathrm{~cm}$ dilatation was done. Arrhythmia was present in all 10 patients with preoperative atrial fibrillation and thrombus was present in 2 patients which did not prohibit CMC procedure (Table-II).

Table-1

Pre-operative Variables

\begin{tabular}{llcc}
\hline Variables & Subclass of variables & Male (n=8) & Female (n=17) \\
\hline Echo finding & Ejection fraction & $51.57 \%$ & $60.27 \%$ \\
& MVA & $0.778 \mathrm{~cm}^{2}$ & $0.943 \mathrm{~cm}^{2}$ \\
& LA size & $50 \mathrm{~mm}$ & $55 \mathrm{~mm}$ \\
& Thrombus in LA appendage & 0 & 2 \\
Arrhythmia & AF & 2 & 8 \\
Anti-arrhythmic drug & Digoxin & 2 & 8 \\
Risk factors & Pregnancy & 0 & 1 \\
& older age at commissuruotomy $(>40$ years) & 1 & 4 \\
NYHA grading & Pulmonary hypertension (> 30 mm of hg) & 4 & 10 \\
& 4 & $45 \%$ & $50 \%$ \\
& 3 & $45 \%$ & $40 \%$ \\
\hline
\end{tabular}

Table-II

Per-operative findings:

\begin{tabular}{lcc}
\hline Variables & Male $(\mathrm{n}=8)$ & Female $(\mathrm{n}=17)$ \\
\hline Dilatation & $3.50 \mathrm{~cm}$ & $3.25 \mathrm{~cm}$ \\
Arrhythmia & 2 & 8 \\
Thrombus in the LA & 0 & 2 \\
appendage & & \\
\hline
\end{tabular}

Atrial fibrillation persisted in all the 10 patients with preoperative history of chronic atrial fibrillation, however, 1 patient suffered from bradycardia and another patient had premature ventricular contraction which revert back to normal after drug therapy. One female had an attack of non-fatal cerebrovascular accident and another aged male patient (age -50 years) died of acute left ventricular failure. 
Table-III

Post-operative complications

\begin{tabular}{llcc}
\hline Variables & $\begin{array}{l}\text { Subclass of } \\
\text { variables }\end{array}$ & $\begin{array}{c}\text { Male } \\
(\mathrm{n}=8)\end{array}$ & $\begin{array}{c}\text { Female } \\
(\mathrm{n}=17)\end{array}$ \\
\hline Arrhythmia & AF & 2 & 8 \\
& Bradycardia & 1 & 0 \\
& PVC & 0 & 1 \\
BP & & 90 (mean) & 85 (mean) \\
SPO2 & $100 \%$ & $99 \%$ \\
Mitral regurgitation & 00 & 00 \\
Reopening & & 00 & 00 \\
Stroke & 00 & 01 \\
Mortality & & 01 & 00 \\
\hline
\end{tabular}

\section{Discussion:}

Usually mitral valve disease has a rheumatic origin and a gradually worsening prognosis if not treated in proper time. It is still a major cause of cardiovascular complications in developing countries. It is estimated that 15.6 million people suffer from rheumatic heart disease world wide, with approximately 282,000 new cases and 233,000 related deaths each year. ${ }^{4}$ The inflammatory deposit on valve endothelium causes inflammation, narrowing, stenosis, leaflet immobility and calcification when it becomes chronic. It gives a downtrend impact on the haemodynamic functions of the cardiac chambers. The natural course of the disease turns it to present as a low fixed cardiac output condition. It produces symptoms and sometimes lifethreatening conditions like uncontrolled arrhythmia, thromboembolic manifestation and heart failure develops which needs aggressive management. Medical management can deal with only the relief of symptoms but cure of the disease depends on some invasive procedures (PTMC/ Balloon valvotomy) or surgery (CMC, OMC valve repair or replacement) depending on the grading of the disease.

The costs of heart operations and the problems related to anticoagulation after prosthetic valve replacement are among the limitations faced by patients in nonindustrialized countries where mitral stenosis is caused by chronic rheumatic heart disease. ${ }^{5}$ The least costly and optimal way to achieve this objective is by closed mitral valvotomy or commissurotomy. ${ }^{5}$ Closed mitral commissurutomy in expert hand is not an unsafe procedure and some studies commented about closed mitral commissurotomy as a safe alternative to open mitral commissurotomy ${ }^{6}$ and balloon mitral commissurutomy
(BMC) in selected patients. Differences of opinion exist and some commented that technical advances had decreased the risk of cardio-pulmonary bypass to the point that closed mitral commissurotomy was performed infrequently in most cardiac centers and was considered hazardous. ${ }^{7}$ Risk factors counted as such that older age at commissuruotomy, higher PVR, mitral leaflet calcification, LV enlargement, post-commissurotomy mitral regurgitation may turn the outcome to a grave one. ${ }^{2}$ Most of the reference studies covered the cases of CMC along a long period of follow up (35, 21, 25, and 14 with a mean of 23.75 years).The total of the number of the cases were 2500 over these period altogether.

In this study, we covered our study cases done between February ' 09 to September ' 09 , the number of cases being only 25 because most other cases required valve replacement procedure. Patients for CMC were selected by stringent criteria and outcome was observed in the immediate post-operative period during the 3-5 day stay in the cardiac ICU. Majority of patients were less than 40 years of age having preserved LV function though having tight mitral stenosis, 10 patients had chronic atrial fibrillation which persisted after surgery, 14 patients had pulmonary hypertension and although the resting global ejection fractions were near to normal their exercise tolerance capacity were very poor ( $90 \%$ patients were NYHA class 3 \& 4) ( Table-1). Dilatations were done up to $3.50 \mathrm{~cm}$ in male and in females up to $3.25 \mathrm{~cm}$ considering their lower body surface area (Table-2). During operation haemodynamic stability was maintained and bradycardia and PVC occurred in only 2 cases. Only 1 patient had episode of postoperative stroke and 1 patient died of uncontrollable left ventricular failure (Table-III).

In some centre, they found the post-operative complication in $3 \%$, operative mortality was $2 \%$, and follow up revealed re-stenosis in $6 \%$, MR in $14 \%$, complications in $7 \%$, late deaths in $3 \%$, re-operation required in $32 \%$ ( 44 of 139 cases) and the follow up was done over 25 years. ${ }^{8}$ They also found improvement in NYHA classification in $82 \%$ of these patients. In another centre in Munich, Germany, survival rates after 10,20 and 30 years were $89 \%, 67.8 \%$ and $49.1 \%$ in group $\mathrm{A}$ (CMC patients) and $91.7 \%, 66.7 \%$ and $45.9 \%$ in group B (OMC patients) which does not have any statistically significant difference between the two groups. ${ }^{9}$ However, in one seven year long study, the researchers claimed that the good results, lower cost, and elimination of drawbacks of thoracotomy and cardiopulmonary bypass indicate that CMC should be the treatment of choice for patients with tight pliable rheumatic MS. ${ }^{10}$ 
In this study, follow up was done only in the immediate post-operative period and found that the outcomes are quite acceptable.

Risk factors were present such as older age at commissuruotomy, higher PVR, mitral leaflet calcification, LV enlargement, post-commissurotomy mitral regurgitation, pregnancy in some of the cases (Table I). In our centre, number of OMC is less and those cases with indication were selected for mitral valve replacement. But considering the financial status of some of the patients, we had to take risk of older age at commissurotomy or very younger age patients where we had to preserve the valves for future replacement. The outcome is reasonably acceptable in selected patients and complies with another study which concludes that' Surgical commissurotomies provide equivalent immediate and long term outcome results and delay the need for mitral valve replacement. ${ }^{4}$

\section{Conclusion:}

Closed mitral commissurutomy is quite acceptable having low morbidity and mortality in expert hand and could be an interim procedure. The number of cases is low so it would be premature to come to a definitive conclusion. However, this limitation of this study could be overcome if it can be done as a cohort study for few years and in multiple centers.

\section{References:}

1. Robert M.Bojar, Kenneth G. Warner; Mannual of perioperative care in cardiac surgery; Chapter I,P-20, $3^{\text {rd }}$ Edition, 1999; Blackwell publishing Ltd.,UK; ISBN 0-632-04365-2.

2. MS Hickey, EH Blackstone, JW Kirklin \& LS Dean; Department of Surgery, University of Alabama, Birmingham School of Medicine 35294; Outcome probabilities and life history after surgical mitral commissurotomy: implications for ballon commissurotomy; J Am Coll Cardiol,1991;17:29-42.
3. Choudhary SK, Dhareshwar J, Govil A, Airan B, Kumar AS; Open mitral commissurotomy in the current era: indiactions, technique, and results; Ann Thorac Surg. 2003 Jan; 75 (I): 41-46.

4. Ronan j. Curtin, Brian P Griffin; Mitral Valve disease: stenosis and regurgitation:http://www.clevelandclinicmeded.com/ medicalpubs/diseasemanagement/cardiology/mitral-valvedisease.

5. R.K.SuriFAMS,FIACS, R. Pathania Mch, N.K. Jha Mch, DNB, H.SinghMch, R.S.Dhaliwal Mch,MNAMS, S.S.Rana Mch, S.K. Thingnam Mch, V. Sarwal Mch and J.S. Gujral FRCS; Closed mitral valvotomy for mitral restenosis: Experience in 113 consecutive cases; J Thorac Cardiovasc Surg 1996;112:727-30.

6. Ates A, Unlu Y,Yekeler I,Erkut B,Balci AY, Ozyazicioglu A, Kocak H.Department of Cardiovascular surgery, Ataturk University School of Medicine, Erzurum, Turkey; Role of closed mitral commissurotomy for mitral stenosis: mid- and long-term surgical outcome of 36 patients; PMID:15769718.

7. W G Attman,S El Tahan,Deopartment of Cardiac Surgery, Faculty of Medicine, Alexandra University,Egypt; Minimally invasive closed mitral commissurotomy; Tex Heart InstJ.1999;26(4): 269-74.

8. Salerno TA,Neilson IR, Charrette EJ, LynnRB; A 25 year experience with the closed method of treatment in 139 patients with mitral stenosis; Ann Thorac Surg. 1981 Apr;31(4):300-4.PMID:10616986

9. Detter C, Fischlein T,Feldmeir C, Nollert G, Reichenspurner H, Reichart B;Department of Cardiac Surgery,Klinikum Grosshadern,Ludwig-Maximilians- university, Munich, Germany; Mitral commissurotomy, a technique outdated? Long-term follow-up over a period of 35 years; Ann Thorac Surg. 1999 Dec; 68(6):2112-28.

10. Mohamed Ben Farhat; Mokdad Ayari; Faouzi Maatouk; Fethi Betbout; Habib Gamra; Mourad Jarrar; Mabrouk Tiss; Sonia Hammami; Rafik Thaalbi; ; Faouzi Addad: Percutaneous Balloon Versus Surgical Closed and Open Mitral Commissurotomy :seven-Year Follow-up Results of a Randomized Trial (From the Division of Cardiology, Department of Medicine, Fattouma Bourguiba University Hospital, Tunisia.); (Circulation. 1998;97:245-50. 